\title{
Acta
Biochimica
Polonica
}

Vol. 47 No. 1/2000

QUARTERLY

\section{A simple model for predicting the free energy of binding be- tween anthracycline antibiotics and $\mathrm{DNA}^{\star}$}

\author{
Witold R. Rudnicki ${ }^{1}$, Małgorzata Kurzepa ${ }^{2}$, Teresa Szczepanik $^{2}$, Waldemar Priebe ${ }^{3}$ \\ and Bogdan Lesyng ${ }^{1,2 凶}$ \\ ${ }^{1}$ Interdisciplinary Centre for Mathematical and Computational Modelling, Warsaw University, \\ A. Pawińskiego 5A, 02-106 Warszawa, Poland; ${ }^{2}$ Department of Biophysics, Warsaw University, \\ F. Żwirki i S. Wigury 93, 02-089 Warszawa, Poland; ${ }^{3}$ The University of Texas M. D. Anderson \\ Cancer Center, Houston, TX 77030, U.S.A.
}

Received: 25 October, 1999

Key words: DNA, anthracyclines, modelling, free energy

\begin{abstract}
A theoretical model for predicting the free energy of binding between anthracycline antibiotics and DNA was developed using the electron density functional (DFT) and molecular mechanics (MM) methods. Partial DFT-ESP charges were used in calculating the MM binding energies for complexes formed between anthracycline antibiotics and oligodeoxynucleotides. These energies were then compared with experimental binding free energies. The good correlation between the experimental and theoretical energies allowed us to propose a model for predicting the binding free energy for derivatives of anthracycline antibiotics and for quickly screening new anthracycline derivatives.
\end{abstract}

Anthracycline antibiotics are widely used in chemotherapy. The parent compounds of this group - daunorubicin (daunomycin) and doxorubicin (adriamycin) - are popular agents used in clinical treatments of cancer (Priebe, 1995; Weiss, 1992; Arcamone \&

\footnotetext{
${ }^{\star}$ Presented at the $7^{\text {th }}$ International Symposium on Molecular Aspects of Chemotheraphy, September 8-11, 1999, Gdańsk, Poland.

All simulations were carried out at ICM, Warsaw University. MSI software was available thanks to the countrywide academic license, funded partially by KBN. B.L. and W.R. were supported by KBN (grant 8T11F01616), W.P. by a grant from the Texas Higher Education Coordinating Board (ATP\#00015-090), and M.K. and T.S. by the Warsaw University, Biophysics Department (BST 622/BF).

${ }^{\circledR}$ To whom correspondence should be addressed; tel: (48 22) 874 9100; fax: (48 22) 874 9115; e-mail: lesyng@icm.edu.pl
}

Abbreviations: ESP, electrostatic potential; DFT, density functional theory; MM, molecular mechanics; MD, molecular dynamics; RMSD, root mean square deviation; PDB, Protein Data Bank; PB, Poisson-Boltzmann. 
Cassinelli, 1998). In addition, the anthracyclines are arguably the best-characterized group of DNA intercalators. Numerous high-resolution crystallographic and NMR structures of daunorubicin and other anthracyclines bound to DNA have been reported (Chaires, 1996). The DNA sequence preference of daunorubicin binding to DNA has been characterized by thermodynamic, kinetic, and footprinting data (for reviews see Chaires, 1990; 1995a; 1995b). Yet, despite the intense efforts being made to synthesize more efficacious anthracyclines with fewer toxic side effects, doxorubicin remain the best chemotherapeutic agent of this class. In fact, it is becoming clear that the rational design of new anthracycline derivatives will require, in addition to structural information (see, e.g., Chaires et al., 1997; Lampidis et al., 1997; Priebe \& Perez-Soler, 1993; Priebe et al., 1995), the development of new theoretical models.

Some groups have already begun working on such models. One recent study devoted to the estimation of the free energy of binding of anthracyclines with DNA (Bagiński et al., 1997) produced two interesting results: (1) the design of a thermodynamic cycle involving unbound and bound states of anthracyclines and (2) the determination of the free energy of binding as the sum of free energy changes along the path of the cycle. Although the theoretical and experimental results were in very good agreement, the reported approach is computationally intensive and very sensitive to the selection of some parameters of the model (see Bagiński et al., 1997); in short, the total binding free energy is the sum of more than 10 individual contributions of both signs whose absolute value reaches $30-50 \mathrm{kcal} /$ mole, which in turn is almost 1 order of magnitude higher than the final result. More recently, a free energy component analysis has been used to model protein-DNA complexes (Jayaram et al., 1999). However, while this approach provides a good physical background for theoretical computations of the binding free energy, its practical application would be limited when comparing larger number of ligands.

In this light, the purpose of the present study was to formulate a method for a quick, practical analysis of anthracyclines derived from a common lead compound and of strongly related ligands having similar binding modes. In conducting our study, we followed Hermans et al. (1999) in assuming that the free energy of binding $\Delta G$ can be conceptually divided into the following four components:

$\Delta G=\Delta G_{\mathrm{i}}+\Delta G_{\mathrm{S}}+\Delta G_{\mathrm{c}}+\Delta G_{\mathrm{a}}$, where

$\Delta G_{\mathrm{i}}$ - represents direct ligand-macromolecule interactions involving Van der Waals and Coulomb interaction energy of ligand atoms with atoms of the macromolecule;

$\Delta G_{\mathrm{S}}$ - is the difference between the solvation energy of the macromolecule and the ligand in complex and in isolation;

$\Delta G_{\mathrm{c}}$ - represents the confinement contribution in the bound state, the ligand being confined to a limited range of orientations and a small volume,

$\Delta G_{\mathrm{a}}-$ represents conformational adjustments (i.e., adjustments in the conformations of the ligand and the macromolecule made to meet requirements of the bound state).

Although this decomposition is approximate, it is useful in aiding the understanding of the differences in binding among various compounds. The first and the fourth component can be determined using MM and/or MD methods. In the case of closely related compounds, the second and third components can be accounted for indirectly on the basis of the following qualitative analysis. The solvation component consists of two terms - the first one is proportional to the change of the total solvent accessible area, and the second one arises from the reorganization of the ionic density around the target molecule. Any change in the total solvent-accessible area is proportional to the area of contact between the target and the ligand, and the shape of the ionic cloud should not be to a large extent dis- 
turbed by the change of the ligand details. Since the non-bonded component will be roughly proportional to the surface of molecular contacts, it is reasonable to assume that the solvation component energy of binding will be correlated with the non-bonded energy. The confinement term can be neglected since it is very similar for closely related ligands having the same binding mode.

If all these assumptions are valid, then the free energy of binding of anthracyclines should be linearly correlated with the energy of binding computed by the MM method. With this in mind, we devised an MM-based model for calculating the free energy of binding between anthracycline antibiotics and DNA, and used it to calculate the free energies of binding for a series of previously reported as well as novel anthracycline derivatives.

\section{MATERIALS AND METHODS}

Using the MM method, we computed the binding energies of a DNA octamer with molecules from a set of anthracycline antibiotics for which the experimental free energies of binding were previously reported (Chaires et al., 1995). We also computed binding energies with this DNA octamer for a set of novel mono- and bis-intercalating compounds based on daunorubicin and doxorubicin (Figs. 1 and 2, respectively). Three-dimensional (3-D) models of all the anthracyclines used were built using an Insight II visual interface (MSI, 1997a) (Fig. 3) to manipulate the experimental structure of bis-daunorubicin (WP631) bound to 5'-d(ACGTACGT)-3' (Robinson et al., 1997), PDB ID: 1AL9 (Bernstein et al., 1977; Sussman et al., 1998). The same octamer was used in the computations. In the case of monointercalating compounds, 3-D structures were generated, first by modeling the WP631 structure and then combining two symmetrically bound molecules. To ensure consistency of the computed energies, the sin- gle molecular structure was used as the reference starting structure.

The geometries of all structures were optimized using density functional theory (DFT) as implemented in the Dmol program (MSI, 1996). The atomic partial charges on the anthracycline antibiotics, reproducing the molecular electrostatic potential, so-called ESP- charges, were computed. All calculations were performed on a fine mesh, with the local VWN functional using the DNP basis set without frozen orbitals.

All MM calculations were carried out using the Amber force-field (Weiner et al., 1984; 1986) as implemented in InsightII release 97.0. An implicit solvent model with the distance dependent "dielectric constant" $\varepsilon=\mathrm{r}$ and reduced charges on the DNA phosphate groups was applied (see Rudnicki \& Lesyng, 1995; 1997). The Discover 2.97 program (MSI, 1997b) was used to calculate the energy minimizations. For an overview of the theoretical methods applied see Lesyng \& McCammon (1993). In the case of monointercalating molecules, the binding energy per molecule was computed using the formula:

$\Delta E=\frac{1}{2}\left(E_{\text {complex }}-2 E_{\mathrm{AA}}-E_{\mathrm{DNA}}\right)$

The coefficient $1 / 2$ appears in this equation because there are two antibiotics bound to each DNA molecule. In the case of bisintercalating molecules, the binding energy per molecule was computed using the formula:

$\Delta E=E_{\text {complex }}-E_{\mathrm{AA}}-E_{\mathrm{DNA}}$

All MM energies were obtained for the optimized structures, with all energy gradients lower than $0.1 \mathrm{kcal} /($ mole $\AA$ ).

Because DNA without an explicit solvent is too flexible, we therefore applied an auxiliary potential restraining DNA heavy atoms close to their initial, experimental positions. We 

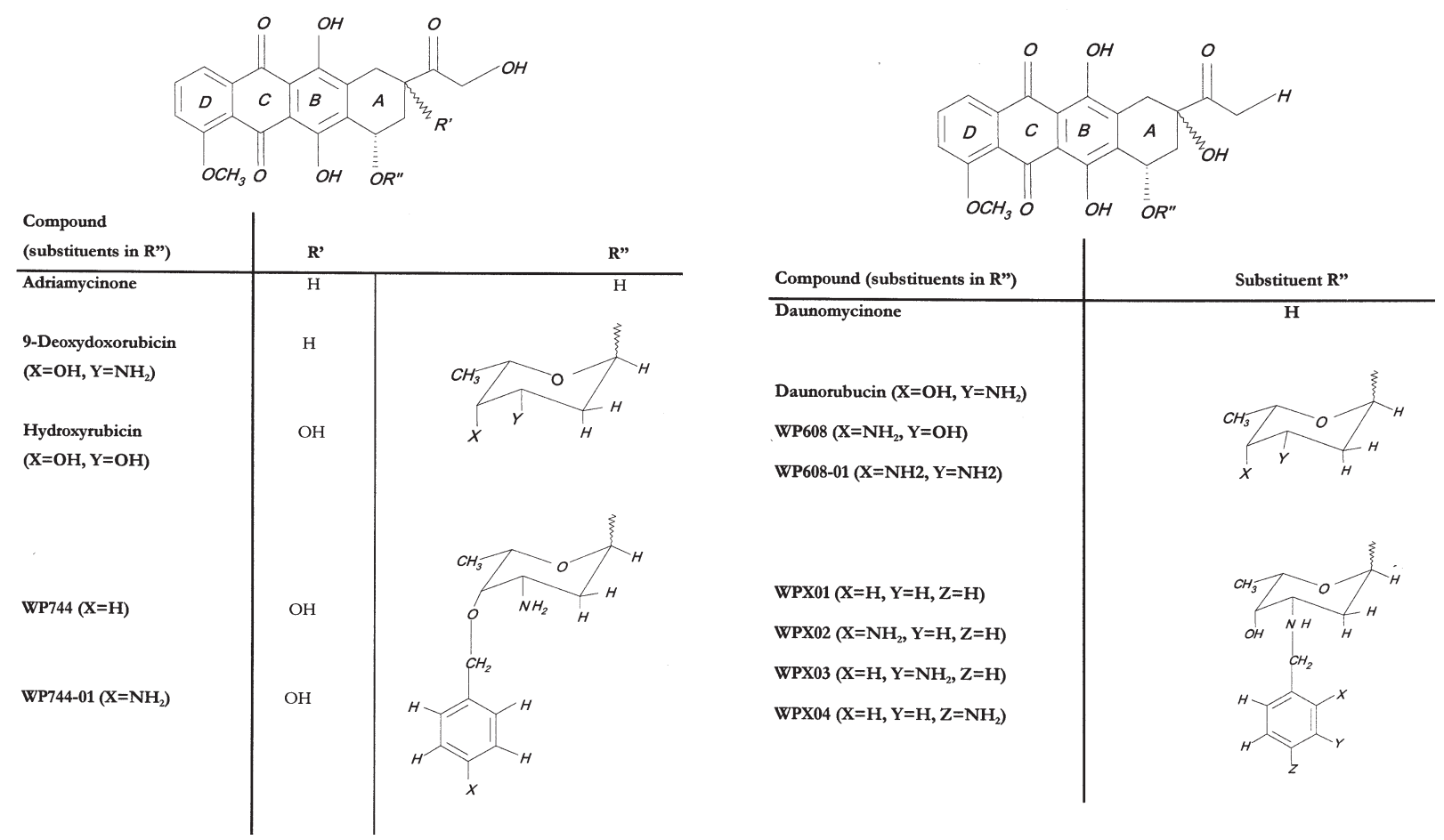

Figure 1. Schematic representation of monointercalating derivatives of daunorubicin a) and doxorubicin b).

All derivatives exhibit the same chiral properties as the original compounds.

then tested each molecule at four different force constants: $100 \mathrm{kcal} /\left(\right.$ mole $\left.\AA^{2}\right), 10 \mathrm{kcal} /$ $\left(\right.$ mole $\left.\AA^{2}\right), 1 \mathrm{kcal} /\left(\right.$ mole $\left.\AA^{2}\right)$, and $0 \mathrm{kcal} /($ mole $\AA^{2}$ ), the latter constant being one of the unconstrained optimization. The theoretical binding energies were then compared with the experimentally derived free energies of binding using a linear regression procedure. We compared the root mean square deviation (RMSD) between the initial and optimized structures, the correlation between the theoretical and experimental energy values, and the difference between the free energies of binding obtained experimentally and from our model.

The straightforward procedure described above required only one computationally intensive step - the computation of the atomic ESP charges of the relatively large drug molecules. But it was desirable to simplify the procedure even further by either computing the ESP charges more quickly (i.e., by minimizing the geometry using a fast force field minimizer and performing a single-point DFT energy calculation to find the ESP charges) or using a simpler procedure to obtain partial charges (i.e., by computing atomic charges using a heuristic algorithm). Opting for the second, simpler approach, we applied the charge equilibration method (Rappe \& Goddard, 1991) implemented in Cerius 2 release 3.8 (MSI, 1998) and then compared the results with the results obtained using the DFT-ESP charges.

\section{RESULTS AND DISCUSSION}

Energy minimization using different force constant values, $k$, of the potential restraining the DNA structure showed that the largest $k$ leads to the best correlation between the experimental free energies of binding and the theoretical ones (Table 1). Conversely, 


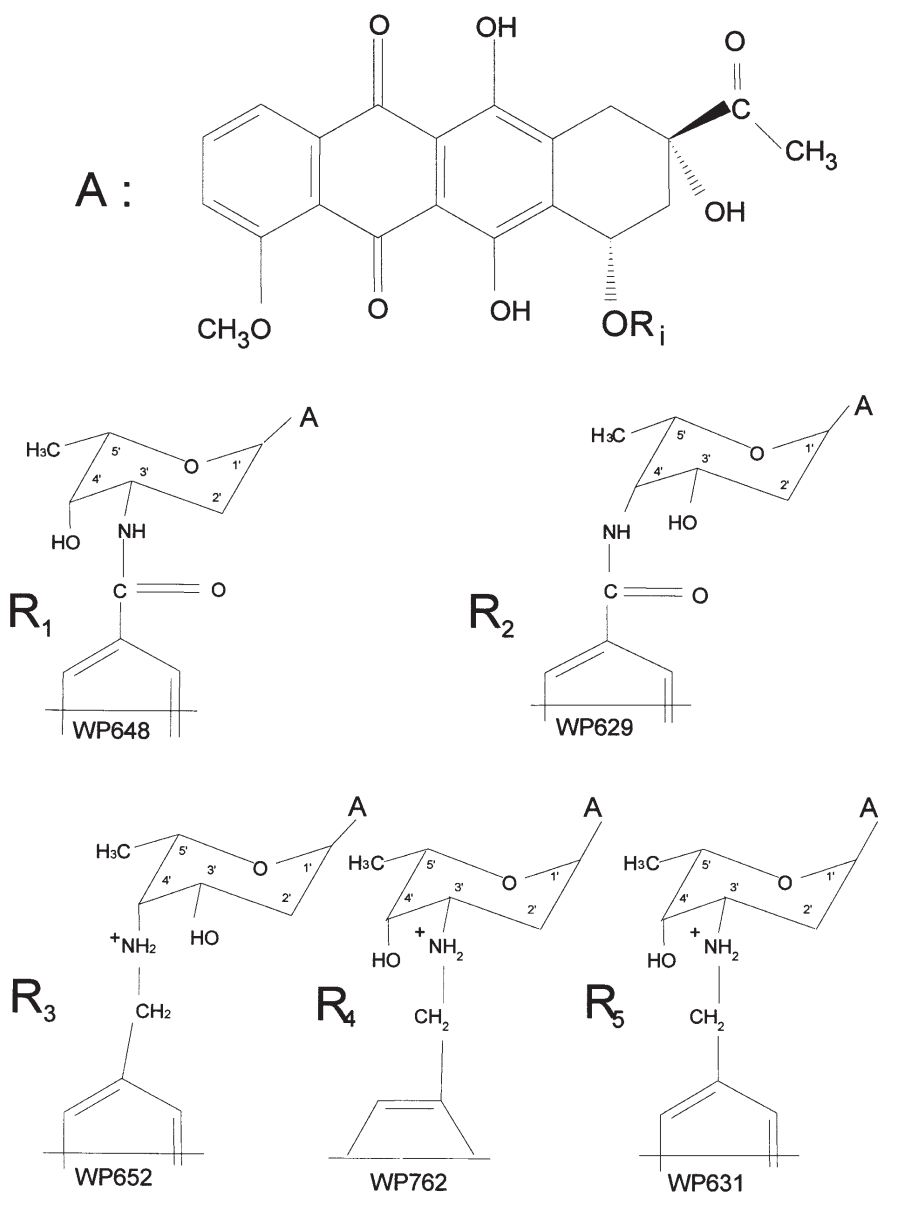

Figure 2. Schematic representation of bisintercalating derivatives of anthracycline antibiotics.

WP 631 is the basic compund described in (Robinson et al., 1997) and the derivatives represent the same chiral symmetry. Only a half of the symmetrical structure is presented. smaller restraining potentials lead to a significantly higher RMSD between the minimized and experimental coordinates and a weaker correlation between the predicted and the experimental energy. It is worth noting, however, that building a model with good correlation between the computed binding energy and the experimental free energy of binding does not require very good description of the electrostatic interactions. Looking at calculations carried out using the partial charges, obtained by the heuristic procedure (Table 1, column E), one may notice that the correlation between the experimental and the computed energy obtained using this charge model and the strong restraining potential is comparable to the correlation obtained using the DFT-ESP charges and the weaker restraining potential.

Table1. Correlations between the experimental free energies of binding $(\Delta G)$ and the theoretical energy of binding $(\Delta E)$ for various models

\begin{tabular}{lccccc}
\hline & A & B & C & D & E \\
Charge model & ESP & ESP & ESP & ESP & Charge equilibration \\
\hline $\begin{array}{l}\text { Restraining potential } \\
\text { force constant } \\
k\left[\mathrm{kcal} /\left(\mathrm{mole}^{2}\right)\right]\end{array}$ & 100 & 10 & 1 & 0 & 100 \\
$\mathrm{r}^{2 *}$ & 0.947 & 0.881 & 0.884 & 0.883 & 0.884 \\
$\mathrm{RMSD}^{* *}[\mathrm{kcal} / \mathrm{mol}]$ & 0.4 & 0.6 & 0.6 & 0.6 & 0.6 \\
\hline
\end{tabular}

${ }^{*}{ }^{2}$ is the correlation coefficient between the theoretical and experimental energies; ${ }^{* *}$ RMSD refers to the theoretical and experimental energies. 


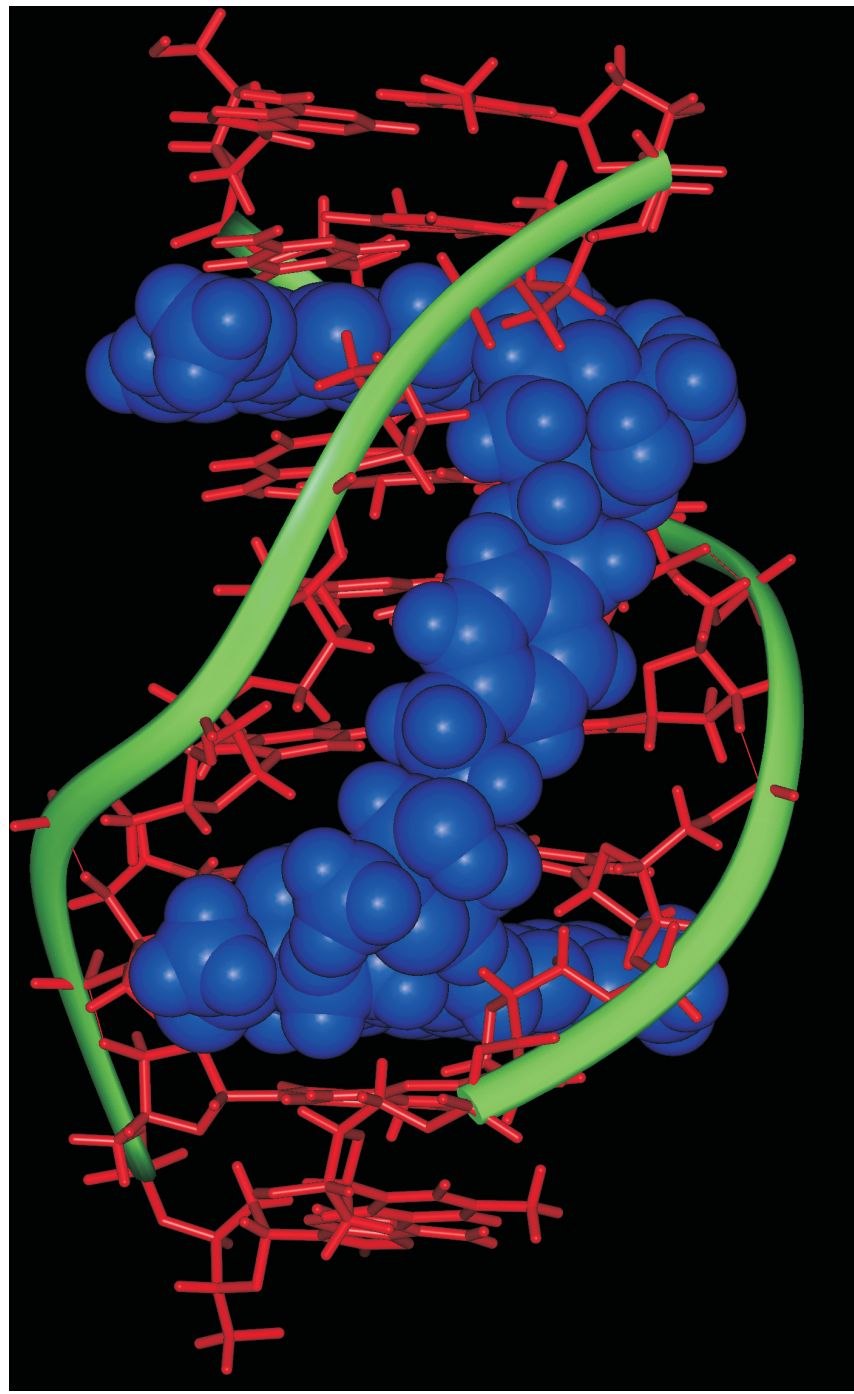

Figure 3. Structure of the bisintercalating anthracycline derivative WP631 in complex with DNA octamer (Robinson et al., 1997). Ribbons represent the phosphate furanose DNA backbones.

When one analyzes the force field binding energies and experimental free energies of binding for a selected set of previously reported anthracycline antibiotics (Table 2), the correlation between the theoretical and experimental values of the binding energies for $k=$
1 (1a) and $k=100 \mathrm{kcal} /\left(\right.$ mole $\AA^{2}$ ) (1b) (Fig. 4), and the free energies of binding for several novel anthracycline derivatives calculated using our model (Table 3), it becomes clear that the binding energies computed using the force field method are much higher than the free en-

Table 2. Theoretical energies of binding $(\Delta E)$, experimental free energies of binding $(\Delta G)$, and predicted theoretical free energies of binding $\left(\Delta G_{\mathrm{T}}\right)$

\begin{tabular}{lccc}
\hline DNA-drug complex & $-\Delta E[\mathrm{kcal} / \mathrm{mol}]$ & $-\Delta G[\mathrm{kcal} / \mathrm{mol}]$ & $-\Delta G_{\mathrm{T}}[\mathrm{kcal} / \mathrm{mol}]$ \\
\hline Daunorubicin & 51.9 & 9.4 & 9.5 \\
Daunomycinone & 33.3 & 5.8 & 5.9 \\
WP608 & 50.3 & 9.2 & 9.2 \\
Doxorubicin & 51.6 & 9.9 & 9.4 \\
Adriamycinone & 33.2 & 6.3 & 5.9 \\
9-Deoxydoxorubicin & 48.5 & 8.8 & 8.8 \\
Hydroxyrubicin & 44.4 & 7.4 & 8.0 \\
\hline
\end{tabular}


ergy of binding for the same components. However, the very clear correlation between these values, which certainly arises from a deeper relation between them, allows reasonably accurate predictions to be made about the free energy of binding. For example, one may notice that the difference between correlations obtained with the DFT-ESP partial charges and with the charges obtained by the equilibration method, is rather small. This shows that the details of the electrostatic interactions are relatively less important for the differentiating interactions between DNA and various anthracycline antibiotics than Van der Waals interactions and solvation. This significant dependence on the restraining potential suggests that a more rigid geometry is required to get a good correlation between the computed and experimental free energies of binding. It is therefore reasonable to assume that the disparity between the computed energy and the experimental free energy of binding for the anthracycline antibiotics can be attributed to interactions with the solvent. In

Table 3. Theoretical energies of binding $(\Delta E)$ and predicted free energies of binding $\left(\Delta G_{\mathrm{T}}\right)$ for novel anthracyclines and DNA

\begin{tabular}{lcc}
\hline \multirow{2}{*}{ DNA-drug complex } & $-\Delta E$ & $-\Delta G_{\mathrm{T}}$ \\
& {$[\mathrm{kcal} / \mathrm{mol}]$} & {$[\mathrm{kcal} / \mathrm{mol}]$} \\
\hline
\end{tabular}

Mono-intercalators

$\begin{array}{lrr}\text { WP608-01 } & 45.5 & 8.3 \\ \text { WP744 } & 58.9 & 10.9 \\ \text { WP744-01 } & 62.9 & 11.8 \\ \text { WPX01 } & 53.4 & 9.8 \\ \text { WPX02 } & 55.5 & 10.3 \\ \text { WPX03 } & 53.8 & 9.9 \\ \text { WPX04 } & 53.1 & 9.8\end{array}$

Bis-intercalators

\begin{tabular}{lll} 
WP631 & 95.5 & 17.3 \\
WP648 & 84.9 & 15.8 \\
WP629 & 62.6 & 11.5 \\
WP762 & 78.7 & 14.6 \\
WP652 & 84.5 & 15.7 \\
\hline
\end{tabular}

fact, it has already been shown that some details of the solvation shell are strongly dependent on the structure of the solute (Rudnicki \& Pettitt, 1997). This dependence can in turn justify the dependence of the correlation between the computed and experimental free energy of binding on the force constant of the restraining potential.

In conclusion, our proposed MM-based approach of linear correlation allows fast, effective, and reasonably accurate estimation of the free energy of binding of new anthracycline derivatives to DNA. This makes our model applicable to fast screening of derivatives of a common lead compound, given a good initial model of interactions between the lead compound and the target molecule, and allows it to be used to select potential drugs on
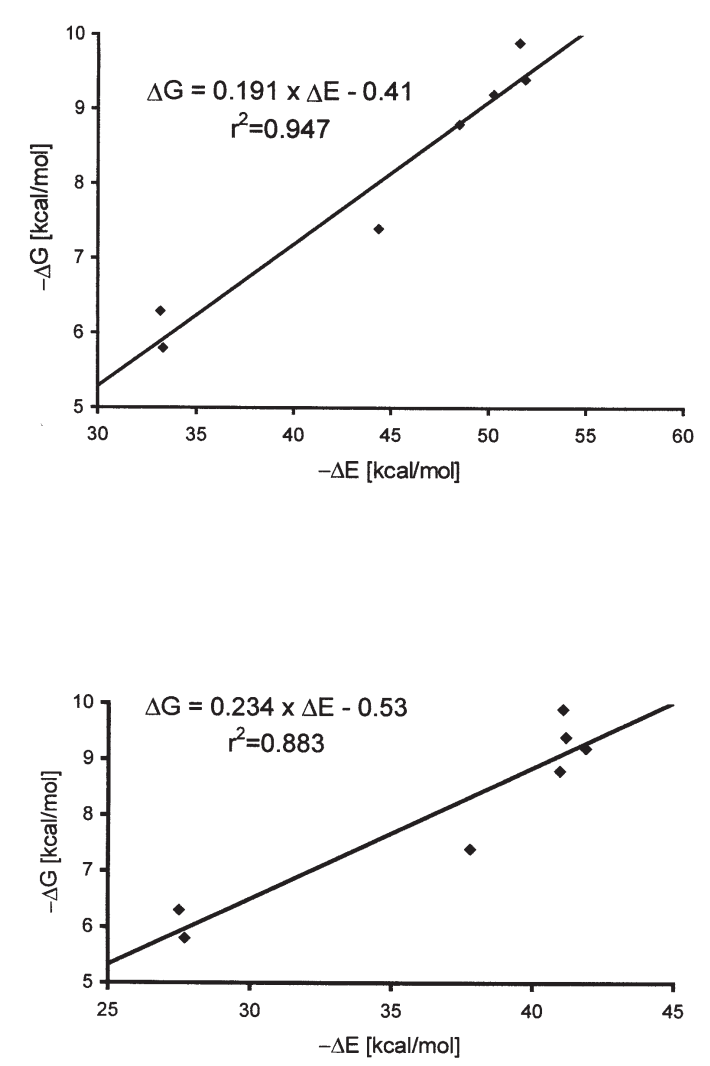

Figure 4. Linear correlation between the theoretical binding energy and the experimental free energy of binding for the experimentally studied anthracycline antibiotics (Chaires et al., 1995). Two restraining force constants were used: (a) $k=100 / \mathrm{kcal} /\left(\mathrm{mol} \AA^{2}\right),(\mathrm{b}) k=0 / \mathrm{kcal} /\left(\operatorname{mol} \AA^{2}\right)-$ no restraints. 
the basis of binding affinity. If the binding of a ligand is comparable to or stronger than the one obtained for daunorubicin and doxorubicin $(\Delta E \approx-9 \mathrm{kcal} / \mathrm{mol})$, then the molecule should be selected for further studies. There is no guarantee, however, that such a ligand will be a better drug, since there are many other factors involved and their influence can only be determin in further studies.

\section{R E F E R E N C E S}

Arcamone, F. \& Cassinelli, G. (1998) Biosynthetic anthracyclines. Curr. Med. Chem. 5, 391-419.

Bagiński, M., Fogolari, F. \& Briggs, J.M. (1997) Electrostatic and non-electrostatic contributions to the binding free energies of anthracycline antibiotics to DNA. J. Mol. Biol. 274, 253-267.

Bernstein, F.C., Koetzle, T.F., Williams, G.J., Meyer E.E., Jr, Brice, M.D., Rodgers, J.R., Kennard, O., Shimanouchi, T. \& Tasumi, M. (1977) The Protein Data Bank: A computer-based archival file for macromolecular structures. J. Mol. Biol. 112, 535-542.

Chaires, J.B. (1990) Biophysical chemistry of the daunomycin-DNA interaction. Biophys. Chem. 35, 191-202.

Chaires, J.B. (1995a) Molecular recognition of DNA by daunorubicin; in Anthracycline Antibiotics: New Analogues, Methods of Delivery, and Mechanisms of Action, ACS Symposium Series 574 (Priebe, W., ed.) pp. 156-167, American Chemical Society, Washington, DC.

Chaires, J.B. (1995b) Daunomycin binding to DNA: From the macrosopic to the microscopic; in Molecular Basis of Specifity in Nucleic Acid-Drug Interactions (Pullman, B. \& Jortner, J., eds.) pp. 123, Kluwer Academic Publishers.

Chaires, J.B., Satyanaraya, S., Dongchul, S., Fokt, I., Przewloka, T. \& Priebe, W. (1995) Parsing the free energy of anthracycline antibiotic binding to DNA. Biochemistry 35, 2047-2053.

Chaires, J.B. (1996) Molecular recognition of DNA by daunorubicin; in Advances in DNA Se- quence Specific Agents 2 (Hurley, L.H. \& Chaires, J.B., eds.) pp. 141-167, JAI Press, Greenwich CT.

Chaires, J.B., Leng, F., Przewloka, T., Fokt, I., Ling, Y.H., Perez-Soler, R. \& Priebe, W. (1997) Structure-based design of a new bisintercalating anthracycline antibiotic. J. Med. Chem. 40, 261-266.

Garrett, A.J.M. \& Poladian, L. (1988) Refined derivation, exact solutions, and singular limits of the Poisson-Boltzmann equation. Ann. Phys. 188, 386-435.

Hermans, J., Mann, G., Wang, L. \& Zhang, L. (1999) Simulation studies of protein-ligand interactions; in Computational Molecular Dynamics: Challenges, Methods, Ideas (Deuflhard, J., Hermans, J., Leimkuhler, B., Mark, A.E., Reich, S. \& Skeel, R.D., eds.) (Lecture Notes in Computational Science and Engineering, 4), pp. 129-148, Springer.

Jayaram, B., McConnell, K.J., Dixit, S.B. \& Beveridge, D.L (1999) Free energy analysis of protein-DNA binding: The EcoRI endonuclease-DNA complex. J. Comp. Phys. 151, 333-357.

Lampidis, T.J., Kolonias, D., Podona, T., Israel, M., Safa, A.R., Lothstein, L., Savaraj, N., Tapiero, H. \& Priebe, W. (1997) Circumvention of P-GP MDR as a function of anthracycline lipophilicity and charge. Biochemistry 36, 2679-2685.

Lesyng, B. \& McCammon, J.A. (1993) Molecular modeling methods. Basic techniques and challenging problems. Pharmac. Ther. 60, 149167.

Molecular Simulations Inc. (1996) DMol, rel. 4.0. San Diego.

Molecular Simulations Inc. (1997a) InsightII, rel 97.0. San Diego.

Molecular Simulations Inc. (1997b) Discover 2.98. San Diego.

Molecular Simulations Inc. (1998) Cerius2 3.8, San Diego.

Priebe, W. \& Perez-Soler, R. (1993) Design and tumor targeting of anthracyclines able to over- 
come multidrug resistance: A double-advantage approach. Pharmac. Ther. 60, 215-234.

Priebe, W. (1995) Anthracycline Antibiotics: New Analogues, Methods of Delivery, and Mechanisms of Action; ACS Symposium Series 574. American Chemical Society, Washington, DC.

Rappe, A.K. \& Goddard, W.A. (1991) Charge equilibration for molecular dynamics simulations. J. Phys. Chem. 95, 3358-3363.

Robinson, H., Priebe, W., Chaires, J.B. \& Wang, A.H.-J. (1997) Binding of two novel bisdaunorubicins to DNA studied by NMR spectroscopy. Biochemistry 36, 8663-8670.

Rudnicki, W.R. \& Lesyng, B. (1995) Applicability of commonly used atom-atom type potential energy functions in structural analysis of nucleic acids. The role of electrostatic interactions. Comp. \& Chem. 19, 253-258.

Rudnicki, W.R. \& Lesyng, B. (1997) Conformational correlations in DNA. Molecular dynamics studies. Molecular Simulation 19, 247-266.
Rudnicki, W.R. \& Pettit, B.M. (1997) Modeling the DNA-solvent interface. Biopolymers 41, 107-119.

Sussman, J.L., Lin, D., Jiang, J., Manning, N.O., Prilusky, J., Ritter, O. \& Abola, E.E. (1998) Protein Data Bank (PDB): Database of three-dimensional structural information of biological macromolecules. Acta Cryst. D54, 1078-1084.

Weiner, S.J., Kollman, P.A., Case, D.A., Singh, U.S., Ghio, C., Alagona, G., Profeta, S., Jr. \& Weiner, P. (1984) A new force field for molecular mechanical simulation of nucleic acids and proteins. J. Am. Chem. Soc. 106, 765-784.

Weiner, S.J., Kollman, P.A., Nguyen, D.T. \& Case, D.A. (1986) An all atom force field for simulations of proteins and nucleic acids. J. Comput. Chem. 7, 230-252.

Weiss, R.B. (1992) The anthracyclines: Will we ever find a better doxorubicin? Semin. Oncol. 19, 670-686. 